\title{
THE MINIMUM TREE FOR A GIVEN ZERO-ENTROPY PERIOD
}

\author{
ESTHER BARRABÉS AND DAVID JUHER
}

Received 5 May 2005 and in revised form 22 September 2005

We answer the following question: given any $n \in \mathbb{N}$, which is the minimum number of endpoints $e_{n}$ of a tree admitting a zero-entropy map $f$ with a periodic orbit of period $n$ ? We prove that $e_{n}=s_{1} s_{2} \cdots s_{k}-\sum_{i=2}^{k} s_{i} s_{i+1} \cdots s_{k}$, where $n=s_{1} s_{2} \cdots s_{k}$ is the decomposition of $n$ into a product of primes such that $s_{i} \leq s_{i+1}$ for $1 \leq i<k$. As a corollary, we get a criterion to decide whether a map $f$ defined on a tree with $e$ endpoints has positive entropy: if $f$ has a periodic orbit of period $m$ with $e_{m}>e$, then the topological entropy of $f$ is positive.

\section{Introduction}

In the last decades, many authors have studied the dynamical behaviour of continuous self-maps of one-dimensional spaces. There are also some books where most of the results are collected in (see, e.g., $[3,7]$ ). In particular, the study of the set of periods of continuous maps $f: X \rightarrow X$, where $X$ is a tree (a graph without circles), has been one of the problems that have centered the attention. The first and most famous result in this direction is Šarkovs'kiu's theorem [13], which gives a complete characterization of the set of periods of $f$ when $X$ is a closed interval of the real line. Baldwin [5] extended this result to the case of an $n$-star (a tree consisting of $n$ edges attached to a unique central point). Recently, Alsedà, Juher, and Mumbrú [2] have developed a characterization of the set of periods of $f$ when $X$ is any generic tree, in terms of the topological structure of $X$ (number and arrangement of vertices, edges, and endpoints of $X$ ).

One way to study the dynamical complexity of a continuous map $f: X \rightarrow X$ of a compact metric space is computing its topological entropy, a nonnegative constant which measures how the iterates of the map mix the points of $X$ (see [1]). It is known that an interval or line map with positive topological entropy is chaotic in the sense of Li and Yorke (see [11]). If $X$ is a general compact metric space, the same result has been recently obtained in [6]. It is also well known that the topological entropy of $f$ is closely related with the sizes of the periodic orbits exhibited by $f$. Some results give upper or lower bounds of the set of periods of $f$ depending on whether $f$ has a positive entropy or not. Of course, these bounds depend strongly on the particular space $X$ under consideration. 
When $X$ is an interval, a map $f: X \rightarrow X$ has a positive entropy if and only if it has a periodic orbit of period different from a power of 2 (several authors have contributed to prove this result, see [3] for a historical survey). The zero-entropy self-maps of a generic tree $X$ have been characterized by Alsedà and Ye [4] by using the notion of division of a periodic orbit (see Section 3 for a definition), which is an important tool in the proof of the main result of this paper. The same authors give a maximal set of periods for zero-entropy maps in terms of the number of endpoints of $X$. Blokh, in [8], proves the following result in the same spirit.

Theorem 1.1 (Blokh). Let $X$ be a tree and let $f: X \rightarrow X$ be continuous. The topological entropy of $f$ is zero if and only if the period of each periodic orbit of $f$ has the form $k \cdot 2^{l}$, where $k$ is odd and not larger than the number of edges of $X$ and all the prime divisors of $k$ are not larger than the number of endpoints of $X$.

Llibre and Misiurewicz, in [12], study the properties of the topological entropy of continuous self-maps of graphs and its relationship with the set of periods. They introduce the notions of "god of a number" and "pantheon of a set of natural numbers". For $k \in \mathbb{N}$, $\operatorname{god}(k)$ denotes the greatest odd divisor of $k$. In other words, if $k=n \cdot 2^{l}$ with $n$ odd and $l \geq 0$, then $\operatorname{god}(k)=n$. For $S \subset \mathbb{N}$, the set $\{\operatorname{god}(k): k \in S\}$ is called the pantheon of $S$. Also, for $k \in \mathbb{N}$, the following number is defined:

$$
\Gamma(k)=\prod_{\substack{3 \leq p \leq 4 k \\ p \text { prime }}}\left[\frac{\log (2 k)}{\log (p / 2)}\right]+1,
$$

where $[\cdot]$ denotes the integer part. Llibre and Misiurewicz show that if $f$ is defined on a graph with $s$ edges and the pantheon of the set of periods of $f$ has more than $s \Gamma(s)$ elements, then the entropy of $f$ is positive. As the authors remark, the estimate $s \Gamma(s)$ is not the best possible one and they have not tried to optimize it. In the case of the interval and the circle, it is known that the best estimate of the minimum number of gods which forces positive entropy is two. But for a generic graph, the problem of determining this minimum remains open.

In this paper we restrict our attention to continuous maps defined on trees. Even in this case, there are still open questions related to the pantheon of the set of periods of a zero-entropy map. The most ambitious problem would be to determine, in terms of the topology of the tree, the maximum number of elements of the pantheon of the set of periods that a zero-entropy map can have. Clearly, this maximum has to be smaller than $s \Gamma(s)$, where $s$ is the number of edges of the tree. Theorem 1.1 gives also an upper bound, much better than $s \Gamma(s)$ in general. One can easily construct simple examples showing that the exact computation of this maximum seems rather difficult and depends not only on the number of endpoints, vertices, and edges of the tree but also on the way they are organized.

There are also many other partial questions and results about the relationship between the geometry of the tree on which a map $f$ is defined, the arithmetics of its set of periods, 
and its topological entropy. One of them deals with the problem of determining the periods forced by the existence of a given periodic orbit. For instance, in [9], it is proved that if the tree has $e$ endpoints and $f$ has an orbit of period larger than $e$, then $f$ has also an orbit of period $m$ for some $1<m \leq e$. See also [10] for similar results.

We are interested in zero-entropy maps defined on trees. Given any $n \in \mathbb{N}$, we determine the minimum $i$ such that there is a tree $T$ with $i$ endpoints and a zero-entropy map $f: T \rightarrow T$ having a periodic orbit of period $n$. From now on, this minimum will be denoted by $e_{n}$. The main result of this paper is the following.

Theorem 1.2. Let $n \in \mathbb{N}$ with $n \geq 2$. Let $n=s_{1} s_{2} \cdots s_{k}$ be the decomposition of $n$ into a product of primes such that $s_{i} \leq s_{i+1}$ for $1 \leq i<k$. Then,

$$
e_{n}=s_{1} s_{2} \cdots s_{k}-\sum_{i=2}^{k} s_{i} s_{i+1} \cdots s_{k} \text {. }
$$

Using the formula given by Theorem 1.2 and a simple inductive argument, it is not difficult to check that $e_{n} \geq 2$ for each $n \geq 2$. Moreover, $e_{n}=2$ if and only if $s_{1}=s_{2}=$ $\cdots=s_{k}=2$.

As a consequence of Theorem 1.2, we obtain the following sufficient condition to decide whether a continuous self-map $f$ defined on a tree $T$ has positive entropy.

Corollary 1.3. Let $f: T \rightarrow T$ be a tree map with a periodic orbit of period $n$. Let e be the number of endpoints of $T$ and let $n=s_{1} s_{2} \cdots s_{k}$ be the decomposition of $n$ into a product of primes such that $s_{i} \leq s_{i+1}$ for $1 \leq i<k$. If

$$
e<s_{1} s_{2} \cdots s_{k}-\sum_{i=2}^{k} s_{i} s_{i+1} \cdots s_{k}
$$

then the topological entropy of $f$ is positive.

For instance, assume that $T$ has 9 endpoints and $f$ is known to have an orbit of period $n=15$. Since $e_{15}=3 \cdot 5-5=10$, it follows that the map $f$ has positive entropy. It is worth observing that this fact cannot be directly obtained from Theorem 1.1. Indeed, $T$ has at most 15 edges (it is well known that any tree with $e$ endpoints has at most $2 e-3$ edges) and, thus, 15 is an admissible period for a zero-entropy map according to Theorem 1.1 since it is odd and is not larger than the number of edges of $T$ and all its prime divisors are not larger than the number of endpoints of $T$.

We also remark that Theorem 1.2, in the same way as the results of Blokh, Llibre, and Misiurewicz, states that the positive entropy of a map $f$ is due to the gods of its periods rather than to the periods themselves. To see it, take $n \in \mathbb{N}$ and set $n^{\prime}=n \cdot 2^{l}$ for some $l \geq 0$. Let $n=s_{1} s_{2} \cdots s_{k}$ and $n^{\prime}=r_{1} r_{2} \cdots r_{m}$ be the decompositions of $n$ and $n^{\prime}$ into a product of primes such that $s_{i} \leq s_{i+1}$ and $r_{i} \leq r_{i+1}$. It is easy to check that

$$
s_{1} s_{2} \cdots s_{k}-\sum_{i=2}^{k} s_{i} s_{i+1} \cdots s_{k}=r_{1} r_{2} \cdots r_{m}-\sum_{i=2}^{m} r_{i} r_{i+1} \cdots r_{m} .
$$


Thus, in view of Theorem 1.2, $e_{n}=e_{n^{\prime}}$. This implies that, for each $n \in \mathbb{N}, e_{n}=e_{\operatorname{god}(n)}$. In other words, $e_{\operatorname{god}(n)}$ endpoints are enough to assure that a zero-entropy map can be defined exhibiting a periodic orbit of period $n$.

\section{Notation and basic definitions}

Given any subset $X$ of a topological space, we will denote by $\operatorname{Int}(X)$ and $\mathrm{Cl}(X)$ the interior and the closure of $X$, respectively. For a finite set $A$, we will denote its cardinality by $|A|$.

By an interval, we mean any space homeomorphic to $[0,1] \subset \mathbb{R}$. A tree is a uniquely arcwise connected space that is either a point or a union of finitely many intervals. Any continuous map from a tree into itself will be called a tree map. The set of periods of all periodic orbits of a tree map $f$ will be called the set of periods of $f$ and will be denoted by $\operatorname{Per}(f)$.

If $T$ is a tree and $x \in T$, we define the valence of $x$ to be the number of connected components of $T \backslash\{x\}$. The valence of $x$ will be denoted by $\operatorname{val}_{T}(x)$ or $\operatorname{simply}$ by val $(x)$. Each point of valence 1 will be called an endpoint of $T$ and the set of such points will be denoted by $\operatorname{En}(T)$. A point of valence different from 2 will be called a vertex of $T$, and the set of vertices of $T$ will be denoted by $V(T)$. The closure of each connected component of $T \backslash V(T)$ will be called an edge of $T$. The number of endpoints and the number of edges of $T$ will be denoted, respectively, by en $(T)$ and ed $(T)$.

Any tree which is a union of $r$ intervals, with $r>1$, whose intersection is a unique point $x$ of valence $r$, will be called an $r$-star, and $x$ will be called its central point.

Given a tree $T$ and $P \subset T$, we will define the convex hull of $P$, denoted by $\langle P\rangle_{T}$ or simply by $\langle P\rangle$, as the smallest closed connected subset of $T$ containing $P$. When $P=\{x, y\}$, we will write $\langle x, y\rangle$ or $[x, y]$ to denote $\langle P\rangle$. The notations $(x, y),(x, y]$, and $[x, y)$ will be understood in the natural way.

The notion of topological entropy, introduced in [1], is defined for continuous maps on compact metric spaces and is a quantitative measure of the dynamical complexity of the map. It is an important topological invariant. The topological entropy of a map $f$ will be denoted by $h(f)$.

\section{Proof of Theorem 1.2}

This section is devoted to prove Theorem 1.2 and, thus, to calculate the constant $e_{n}$ for any $n \in \mathbb{N}$. We recall that this constant has been defined in Section 1 as $e_{n}=\min _{i \in \mathbb{N}}\{$ there is a tree map $f: T \longrightarrow T$ with $\operatorname{en}(T)=i, h(f)=0, n \in \operatorname{Per}(f)\}$.

In order to calculate $e_{n}$, we will use the characterization of the zero-entropy tree maps given in [4]. Next we introduce the necessary notions. Let $f: T \rightarrow T$ be a tree map and let $P$ be a periodic orbit of $f$. The map $f_{P}:\langle P\rangle \rightarrow\langle P\rangle$ defined by $f_{P}=r \circ f$, where $r: T \rightarrow\langle P\rangle$ is the natural retraction, will be called the natural restriction of $f$ to $\langle P\rangle$. Let $y$ be a fixed point of $f_{P}$. We will denote by $Z^{P, y}$ the connected component of $\langle P\rangle \backslash P$ containing $y$, and by $Z_{1}^{P, y}, Z_{2}^{P, y}, \ldots, Z_{l}^{P, y}$ the connected components of $\langle P\rangle \backslash Z^{P, y}$.

Let $P$ be a periodic orbit of a tree map $f: T \rightarrow T$. We say that $P$ has a division if for a fixed point $y$ of $f_{P}$, there exist $\left\{M_{1}, M_{2}, \ldots, M_{m}\right\}$ with $m \geq 2$, a partition of $\langle P\rangle \backslash Z^{P, y}$ such that each $M_{i}$ consists of a union of some of the sets $Z_{j}^{P, y}, f\left(M_{i} \cap P\right)=M_{i+1} \cap P$ 
for $1 \leq i<m$, and $f\left(M_{m} \cap P\right)=M_{1} \cap P$. The sets $M_{i}$ will be called the branches of $P$. Observe that each branch contains $|P| / m$ points of $P$.

Remark 3.1. In the notation used in the definition of division, it is not difficult to check that

(i) $\left\langle M_{i}\right\rangle \cap M_{j}=\varnothing$ whenever $i \neq j$. Hence, $M_{i} \cap P=\left\langle M_{i}\right\rangle \cap P$;

(ii) $\operatorname{En}\left(\left\langle M_{i}\right\rangle\right) \cap \operatorname{En}(\langle P\rangle) \neq \varnothing$;

(iii) there is at most one endpoint of $\left\langle M_{i}\right\rangle$ which is not an endpoint of $\langle P\rangle$. Thus, $\sum_{i=1}^{m} \mathrm{en}\left(\left\langle M_{i}\right\rangle\right)-m \leq \mathrm{en}(\langle P\rangle) \leq \sum_{i=1}^{m} \mathrm{en}\left(\left\langle M_{i}\right\rangle\right)$;

(iv) if $\left|P \cap M_{i}\right|=1$, then $M_{i}$ reduces to the only point of $P \cap M_{i}$. Thus, if each branch contains one point of $P$, then $\sum_{i=1}^{m}$ en $\left(\left\langle M_{i}\right\rangle\right)=\operatorname{en}(\langle P\rangle)=|P|$.

The following result, which is [4, Corollary C], characterizes the zero-entropy tree maps in terms of their orbits.

Theorem 3.2. Let $f: T \rightarrow T$ be a tree map. The following statements are equivalent.

(1) $h(f)=0$.

(2) For every $n \in \mathbb{N}$, each periodic orbit of $f^{n}$ of a period larger than 1 has a division.

(3) $\operatorname{Per}(f) \subset\left\{k \cdot 2^{l}: k \leq \operatorname{en}(T)\right.$ ! odd, and $\left.l \in \mathbb{N} \cup\{0\}\right\}$.

Theorems 3.2 and 1.1 will be the main tools to calculate the number $e_{n}$ in general. However, it is easy to give simple expressions of $e_{n}$ in some particular cases.

Lemma 3.3. Let $n \in \mathbb{N}$.

(1) If $n=1$, then $e_{n}=1$.

(2) If $n=2^{k}$ for some $k \geq 1$, then $e_{n}=2$.

(3) If $n$ is prime, then $e_{n}=n$.

Proof. Statement (1) is obvious. Statement (2) follows from the fact that, given any $k \in$ $\mathbb{N}$, one can define a zero-entropy interval map exhibiting a periodic orbit of period $2^{k}$. Indeed, by Šarkovs'kiı̌'s theorem [13], there exists an interval map $f$ such that $\operatorname{Per}(f)=$ $\left\{1,2,2^{2}, \ldots, 2^{k}\right\}$. By Theorem 1.1, the topological entropy of $f$ is zero.

Now let us prove (3). Let $T$ be a tree with en $(T)=e_{n}$ and let $f: T \rightarrow T$ be a zeroentropy map having a periodic orbit $P$ of period $n$. Without loss of generality, we can assume that $\operatorname{En}(T) \subset P$. Indeed, otherwise the natural restriction of $f$ on $\langle P\rangle$ would be a zero-entropy map having $P$ as a periodic orbit and en $(\langle P\rangle) \leq \mathrm{en}(T)$. Therefore, from now on, we assume that $\langle P\rangle=T$. By Theorem 3.2(2), $P$ has a division. Let $m \geq 2$ be the number of branches of $P$ and let $k$ be the number of points of $P$ in each branch. Since $n=k \cdot m$ and $n$ is prime, it follows that $m=n$ and $k=1$. By Remark 3.1(iv), each $M_{i}$ reduces to the only point of $P \cap M_{i}$ and $e_{n}=\operatorname{en}(\langle P\rangle)=\sum_{i=1}^{m} \operatorname{en}\left(\left\langle M_{i}\right\rangle\right)=m=n$.

Let us introduce a few more notions. Let $\Sigma$ be the set of finite sequences $\left(s_{1}, s_{2}, \ldots, s_{k}\right)$ such that $s_{i} \in \mathbb{N}$ and $s_{i} \geq 2$ for $1 \leq i \leq k$. Let $f: T \rightarrow T$ be a zero-entropy tree map and let $P$ be a periodic orbit of $f$. By Theorem 3.2(2), $P$ has a division. Next we will associate an element of $\Sigma$ to the orbit $P$. This element will be denoted by $\mathbf{s}^{P}$. Let $t_{1} \geq 2$ be the number of branches of $P$ and let $M$ be a branch of $P$. Observe that $P \cap M$ is a periodic orbit of $f^{t_{1}}$ of period $|P| / t_{1}$. If $|P| / t_{1}>1$, then $P \cap M$ has a division by Theorem 3.2(2). Thus, we can repeat the previous argument with $P \cap M$ instead of $P$ and denote the number of 
branches of $P \cap M$ by $t_{2} \geq 2$. By iterating as far as possible, after a finite number of steps, we get a sequence $\left(t_{k}, t_{k-1}, \ldots, t_{2}, t_{1}\right) \in \Sigma$, which we denote by $\mathbf{s}^{P}$. We note that, since the number of branches of a periodic orbit with a division is not uniquely determined, there is not a unique way of performing each step in the construction of $\mathbf{s}^{P}$. Thus, $\mathbf{s}^{P}$ is not uniquely determined. From now on, when we write $\mathbf{s}^{P}=\mathbf{r}$ for a certain $\mathbf{r} \in \Sigma$, we mean that there is a way of doing the above construction which yields $\mathbf{r}$.

For each $\mathbf{s} \in \Sigma$, let us define inductively a tree which will be called an $\mathbf{s}$-star. For $\mathbf{s}=$ $\left(s_{1}\right)$, an $\mathbf{s}$-star is simply an $s_{1}$-star. Let $\mathbf{s}=\left(s_{1}, s_{2}, \ldots, s_{k}\right)$ with $k>1$. Consider an $s_{k}$-star $S$ and $s_{k}$-many copies $T^{1}, T^{2}, \ldots, T^{s_{k}}$ of an $\left(s_{1}, s_{2}, \ldots, s_{k-1}\right)$-star. That is, for $1 \leq i \leq k$, there exists a homeomorphism $h_{i}: T^{i} \rightarrow T^{1}$. Let $x_{1}, x_{2}, \ldots, x_{s_{k}}$ be the endpoints of $S$. Choose an endpoint $w$ of $T^{1}$. An $\mathbf{s}$-star is the tree obtained when one glues together $S, T^{1}, T^{2}, \ldots, T^{s_{k}}$ by identifying $x_{i}$ with $h_{i}(w)$, for each $1 \leq i \leq s_{k}$.

Observe that, since there are several choices for the endpoint $w$ in the construction above, for a given $\mathbf{s} \in \Sigma$, there can be several nonhomeomorphic s-stars.

Remark 3.4. Let $\mathbf{s}=\left(s_{1}, s_{2}, \ldots, s_{k}\right) \in \Sigma$. If $k=1$, then the number of endpoints of an $\mathbf{s}$ star is $s_{1}$. When $k \geq 2$, the number of endpoints of any $\mathbf{s}$-star is $s_{k}(p-1)$, where $p$ is the number of endpoints of any $\left(s_{1}, s_{2}, \ldots, s_{k-1}\right)$-star. An inductive argument easily yields that the number of endpoints of any s-star is

$$
s_{1} s_{2} \cdots s_{k}-\sum_{i=2}^{k} s_{i} s_{i+1} \cdots s_{k} .
$$

Let $\mathbf{s} \in \Sigma$. A tree $T$ will be called $\mathbf{s}$-admissible if there exists a zero-entropy tree map $f: T \rightarrow T$ exhibiting a periodic orbit $P$ such that $\mathbf{s}^{P}=\mathbf{s}$.

Lemma 3.5. For each $\mathbf{s} \in \Sigma$, an $\mathbf{s}$-star is an $\mathbf{s}$-admissible tree.

Proof. We will prove the following claim: there exists a zero-entropy s-star map exhibiting a periodic orbit $P$ such that $\mathbf{s}^{P}=\mathbf{s}$ and the endpoints of the $\mathbf{s}$-star belong to $P$. Write $\mathbf{s}=\left(s_{1}, s_{2}, \ldots, s_{k}\right)$. Let us prove the claim by induction on $k$. For $k=1$, the claim follows by considering a rigid rotation of an $s_{1}$-star $T$ and the orbit composed by the endpoints of $T$.

Now let $k \geq 2$ and assume that the claim holds for $i$ instead of $k$, for each $1 \leq i<k$. Let $R$ be an s-star. By definition, there exists a subtree $S \subset R$ such that $S$ is an $s_{k}$-star and $R \backslash S$ consists of $s_{k}$-many disjoint connected components whose closures $T^{1}, T^{2}, \ldots, T^{s_{k}}$ are pairwise homeomorphic $\left(s_{1}, s_{2}, \ldots, s_{k-1}\right)$-stars. Let $x_{i}$ be the only endpoint of $S$ which belongs to $T^{i}$. For $1 \leq i<s_{k}$, let $\phi_{i}: T^{i} \rightarrow T^{i+1}$ be a homeomorphism such that $\phi_{i}\left(x_{i}\right)=$ $x_{i+1}$ and let $\phi_{s_{k}}: T^{s_{k}} \rightarrow T^{1}$ be a homeomorphism such that $\phi_{s_{k}}\left(x_{s_{k}}\right)=x_{1}$. In addition, we choose the homeomorphisms in such a way that $\phi_{s_{k}} \circ \phi_{s_{k-1}} \circ \cdots \circ \phi_{1}$ is the identity map on $T^{1}$. By the induction hypothesis, there exists a zero-entropy tree map $g: T^{1} \rightarrow T^{1}$ with a periodic orbit $Q$ such that $Q \supset \operatorname{En}\left(T^{1}\right)$ and $\mathbf{s}^{Q}=\left(s_{1}, s_{2}, \ldots, s_{k-1}\right)$. Now consider the map $f: R \rightarrow R$ defined as follows:

(i) for each $1 \leq i<s_{k}$ and each $x \in T^{i}$, set $f(x)=\phi_{i}(x)$;

(ii) for each $x \in T^{s_{k}}$, set $f(x)=g\left(\phi_{s_{k}}(x)\right)$; 
(iii) denote by $y$ the central point of $S$. Set $f(y)=y$. For each $1 \leq i \leq s_{k}$, define $f$ to map $\left[y, x_{i}\right]$ monotonically onto $\left[y, f\left(x_{i}\right)\right]$.

It is not difficult to check that $f$ is well defined and continuous, $\left.f^{s_{k}}\right|_{T^{1}}=g$, and for each $n$-periodic orbit $X$ of $g, \bigcup_{i=0}^{s_{k}} f^{i}(X)$ is an $s_{k} n$-periodic orbit of $f$. Conversely, the period of each periodic orbit $X$ of $f$ (excluding the fixed point $y$ ) has the form $s_{k} n$ for some $n \in \mathbb{N}$, and the restriction of $X$ to $T^{1}$ is an $n$-periodic orbit of $g$. Summarizing, there is a bijection between the set of $n$-periodic orbits of $g$ and the set of $s_{k} n$-periodic orbits of $f$. It easily follows that $h(f)=h(g) / s_{k}$ and therefore $h(f)=0$. Set $P=\bigcup_{i=0}^{s_{k}} f^{i}(Q)$. Then $\operatorname{En}(R) \subset P$ and it is not difficult to see that $\boldsymbol{s}^{P}=\left(s_{1}, s_{2}, \ldots, s_{k}\right)$, since $\mathbf{s}^{Q}=\left(s_{1}, s_{2}, \ldots, s_{k-1}\right)$. This completes the induction step and proves the lemma.

LEMma 3.6. For each $\mathbf{s} \in \Sigma$, an $\mathbf{s}$-star has the minimum number of endpoints among the set of s-admissible trees.

Proof. Set $\mathbf{s}=\left(s_{1}, s_{2}, \ldots, s_{k}\right)$ and let $T$ be an $\mathbf{s}$-admissible tree. That is, there exists a periodic orbit $P$ of a zero-entropy map $f: T \rightarrow T$ such that $\mathbf{s}^{P}=\mathbf{s}$. It is enough to prove that en $(T)$ is not smaller than the number of endpoints of an s-star. To prove this claim, we proceed by induction on $k$. For $k=1$, we have $\mathbf{s}=\left(s_{1}\right)$ and we must see that $T$ has at least $s_{1}$ endpoints. Since $\mathbf{s}^{P}=\left(s_{1}\right), P$ has $s_{1}$ branches and each branch contains exactly one point of $P$. By Remark 3.1(iv), each branch $M_{i}$ reduces to the only point of $P \cap M_{i}$ and $|P|=s_{1}=\operatorname{en}(\langle P\rangle)$. Since en $(\langle P\rangle) \leq \mathrm{en}(T)$, the claim holds for $k=1$.

Now let $k \geq 2$ and assume that the claim holds for $i$ instead of $k$ for each $1 \leq i<k$. Since $\mathbf{s}^{P}=\left(s_{1}, s_{2}, \ldots, s_{k}\right), P$ has $s_{k}$ branches. Choose a branch $M$ of $P$ with the least number of endpoints. By Remark 3.1(iii), we have that

$$
\text { en }(\langle P\rangle) \geq s_{k} \text { en }(\langle M\rangle)-s_{k}=s_{k}(\text { en }(\langle M\rangle)-1) \text {. }
$$

By definition of division, $M \cap P$ is a periodic orbit of $f^{s_{k}}$ of period $|P| / s_{k}$. Moreover, $\mathbf{s}^{M \cap P}=\left(s_{1}, s_{2}, \ldots, s_{k-1}\right)$ since $\mathbf{s}^{P}=\mathbf{s}$. Let $g$ be the natural restriction of $f^{s_{k}}$ to $\langle M\rangle$. By standard results (see, e.g., [3, Lemma 4.3.1(a)]), $h(g) \leq h\left(f^{s_{k}}\right)=s_{k} h(f)=0$. Summarizing, $g:\langle M\rangle \rightarrow\langle M\rangle$ is a zero-entropy tree map with a periodic orbit $M \cap P$ such that $\mathbf{s}^{M \cap P}=\left(s_{1}, s_{2}, \ldots, s_{k-1}\right)$. Let $p$ be the number of endpoints of any $\left(s_{1}, s_{2}, \ldots, s_{k-1}\right)$-star. By the induction hypothesis, en $(\langle M\rangle)$ is not smaller than $p$. Since en $(T) \geq \operatorname{en}(\langle P\rangle)$, from (3.2) it follows that en $(T) \geq s_{k}(p-1)$, which, by Remark 3.4, equals the number of endpoints of an s-star.

LEMmA 3.7. Let $\mathbf{s}=\left(s_{1}, s_{2}, \ldots, s_{k}\right) \in \sum$ with $k \geq 2$ and assume that $s_{p}>s_{p+1}$ for some $p \in$ $\{1,2, \ldots, k-1\}$. Let $\mathbf{s}^{\prime}=\left(s_{1}, s_{2}, \ldots, s_{p-1}, s_{p+1}, s_{p}, s_{p+2}, s_{p+3}, \ldots, s_{k}\right)$. Then the number of endpoints of an $\mathbf{s}^{\prime}$-star is smaller than the number of endpoints of an $\mathbf{s}$-star.

Proof. Observe that, for each $N \in \mathbb{N},\left((N-1) s_{p+1}-1\right) s_{p}<\left((N-1) s_{p}-1\right) s_{p+1}$. The statement follows easily from this fact, Remark 3.4, and a simple inductive argument.

Lemma 3.8. Let $\mathbf{s}=\left(s_{1}, s_{2}, \ldots, s_{k}\right) \in \sum$ and assume that $s_{p}=m \cdot n$, with $n, m \geq 2$, for some $p \in\{1,2, \ldots, k\}$. Let $\mathbf{s}^{\prime}=\left(s_{1}, s_{2}, \ldots, s_{p-1}, m, n, s_{p+1}, s_{p+2}, \ldots, s_{k}\right)$. Then the number of endpoints of an $\mathbf{s}^{\prime}$-star is smaller than the number of endpoints of an $\mathbf{s}$-star. 
3032 The minimum tree for a given zero-entropy period

Proof. Observe that, for each $N \in \mathbb{N},((N-1) m-1) n<(N-1) s_{p}$. The statement follows easily from this fact, Remark 3.4, and a simple inductive argument.

Finally, we are ready to prove Theorem 1.2.

Proof of Theorem 1.2. Let us call any $\mathbf{r}=\left(r_{1}, r_{2}, \ldots, r_{l}\right) \in \sum$ with $n=r_{1} r_{2} \cdots r_{l}$ a decomposition of $n$. In particular, $\mathbf{s}=\left(s_{1}, s_{2}, \ldots, s_{k}\right)$ is a decomposition of $n$. By Lemma 3.6, for each decomposition $\mathbf{r}$ of $n$, an $\mathbf{r}$-star has the minimum number of endpoints among the set of r-admissible trees. Therefore,

$$
e_{n}=\min \{\operatorname{en}(T): T \text { is an } \mathbf{r} \text {-star for a decomposition } \mathbf{r} \text { of } n\} .
$$

Observe that there are only finitely many decompositions of $n$. Using iteratively Lemmas 3.7 and 3.8 finitely many times, one gets that the minimum in the right-hand side of (3.3) is reached when $\mathbf{r}=\mathbf{s}$. Thus, $e_{n}$ is the number of endpoints of an $\mathbf{s}$-star and the theorem follows from (3.1).

\section{Acknowledgment}

The authors have been partially supported by DGES Grants BFM2003-09504-C02-01 and BFM2002-01344.

\section{References}

[1] R. L. Adler, A. G. Konheim, and M. H. McAndrew, Topological entropy, Trans. Amer. Math. Soc. 114 (1965), no. 2, 309-319.

[2] L. Alsedà, D. Juher, and P. Mumbrú, Periodic behavior on trees, Ergodic Theory Dynam. Systems 25 (2005), no. 5, 1373-1400.

[3] L. Alsedà, J. Llibre, and M. Misiurewicz, Combinatorial Dynamics and Entropy in Dimension One, Advanced Series in Nonlinear Dynamics, vol. 5, World Scientific, New Jersey, 2000.

[4] L. Alsedà and X. Ye, No division and the set of periods for tree maps, Ergodic Theory Dynam. Systems 15 (1995), no. 2, 221-237.

[5] S. Baldwin, An extension of Šarkovskiı̌'s theorem to the n-od, Ergodic Theory Dynam. Systems 11 (1991), no. 2, 249-271.

[6] F. Blanchard, E. Glasner, S. Kolyada, and A. Maass, On Li-Yorke pairs, J. reine angew. Math. 547 (2002), 51-68.

[7] L. S. Block and W. A. Coppel, Dynamics in One Dimension, Lecture Notes in Mathematics, vol. 1513, Springer, Berlin, 1992.

[8] A. M. Blokh, Periods implying almost all periods for tree maps, Nonlinearity 5 (1992), no. 6, 1375-1382.

[9] W. Imrich, Periodic points of small periods of continuous mappings of trees, Cycles in Graphs (Burnaby, BC, 1982), North-Holland Math. Stud., vol. 115, North-Holland, Amsterdam, 1985, pp. 443-446.

[10] W. Imrich and R. Kalinowski, Periodic points of continuous mappings of trees, Cycles in Graphs (Burnaby, BC, 1982), North-Holland Math. Stud., vol. 115, North-Holland, Amsterdam, 1985, pp. 447-460.

[11] T. Y. Li and J. A. Yorke, Period three implies chaos, Amer. Math. Monthly 82 (1975), no. 10, 985-992.

[12] J. Llibre and M. Misiurewicz, Horseshoes, entropy and periods for graph maps, Topology 32 (1993), no. 3, 649-664. 
[13] O. M. Šarkovs'kiĭ, Co-existence of cycles of a continuous mapping of the line into itself, Ukrain. Mat. Z̆ 16 (1964), 61-71 (Russian), English translation: Proceedings of the Conference "Thirty Years after Sharkovskii's theorem: New perspectives" (Murcia, 1994). Internat. J. Bifur. Chaos 5 (1995), 1263-1273.

Esther Barrabés: Departament d'Informàtica i Matemàtica Aplicada, Universitat de Girona, Lluís Santaló s/n, 17071 Girona, Spain

E-mail address: barrabes@ima.udg.es

David Juher: Departament d'Informàtica i Matemàtica Aplicada, Universitat de Girona, Lluís Santaló s/n, 17071 Girona, Spain

E-mail address: juher@ima.udg.es 


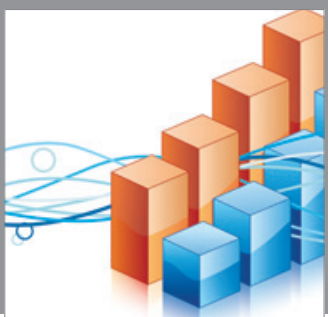

Advances in

Operations Research

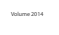

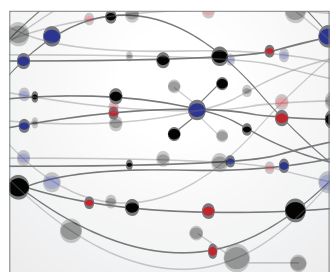

\section{The Scientific} World Journal
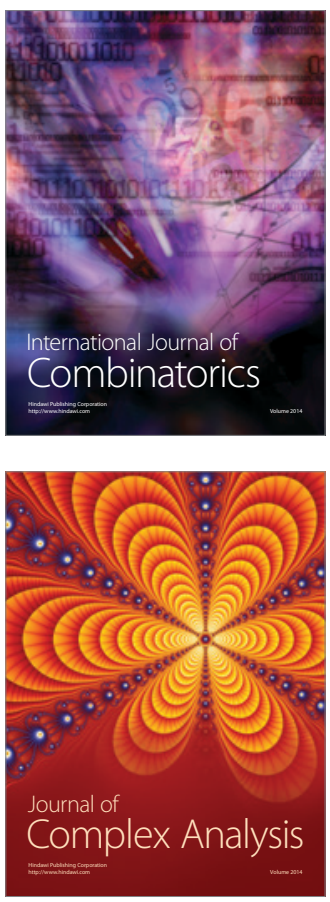

International Journal of

Mathematics and

Mathematical

Sciences
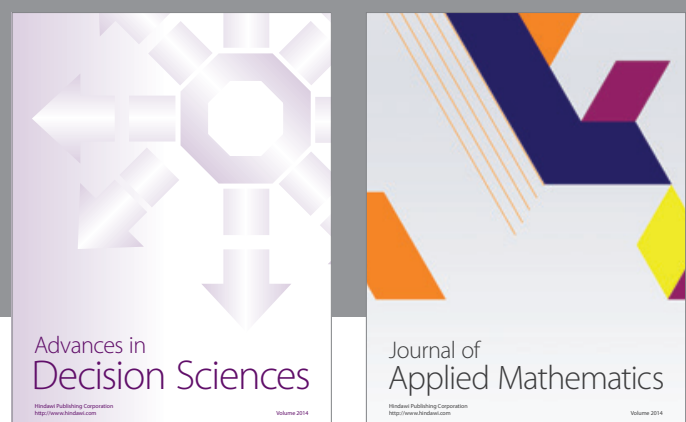

Journal of

Applied Mathematics
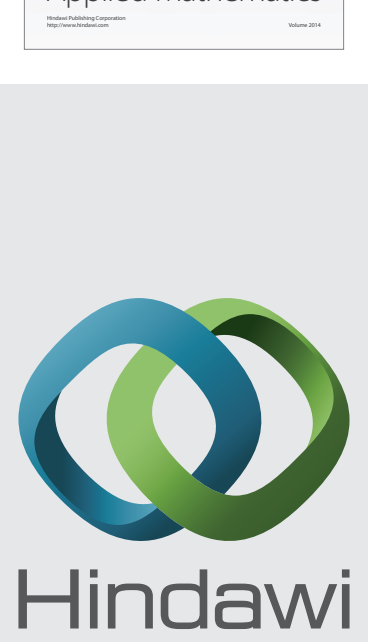

Submit your manuscripts at http://www.hindawi.com
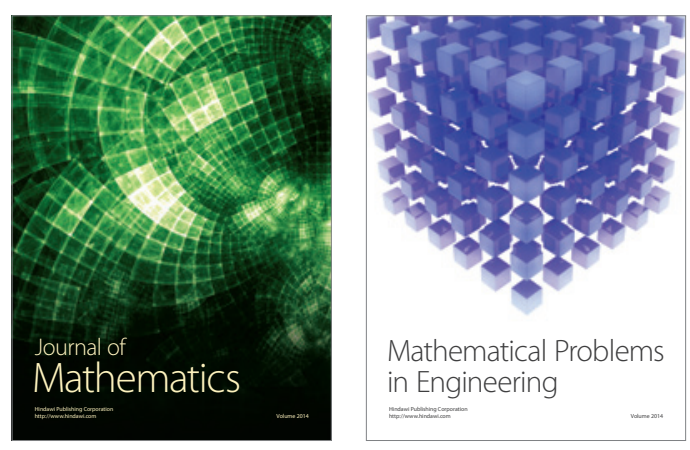

Mathematical Problems in Engineering
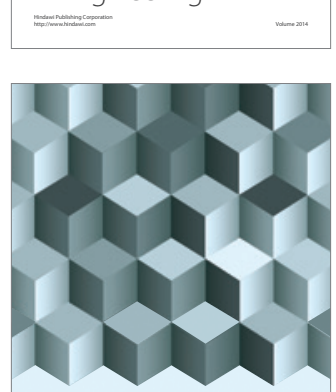

Journal of

Function Spaces
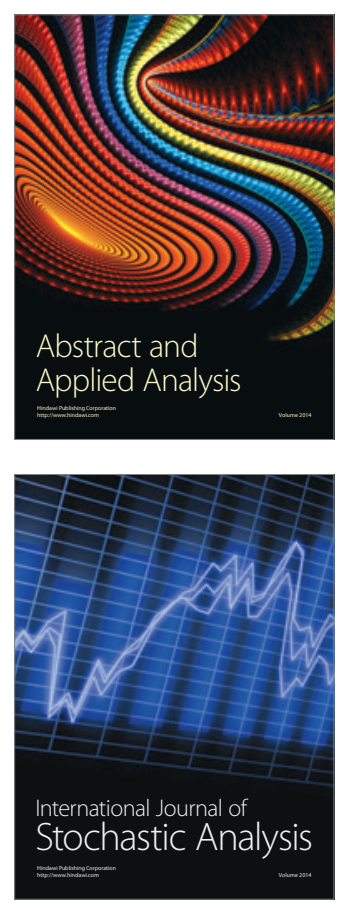

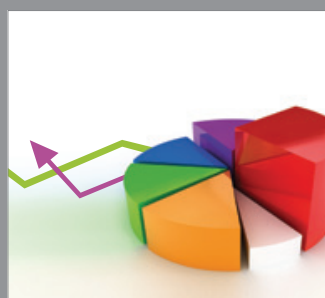

ournal of

Probability and Statistics

Promensencen
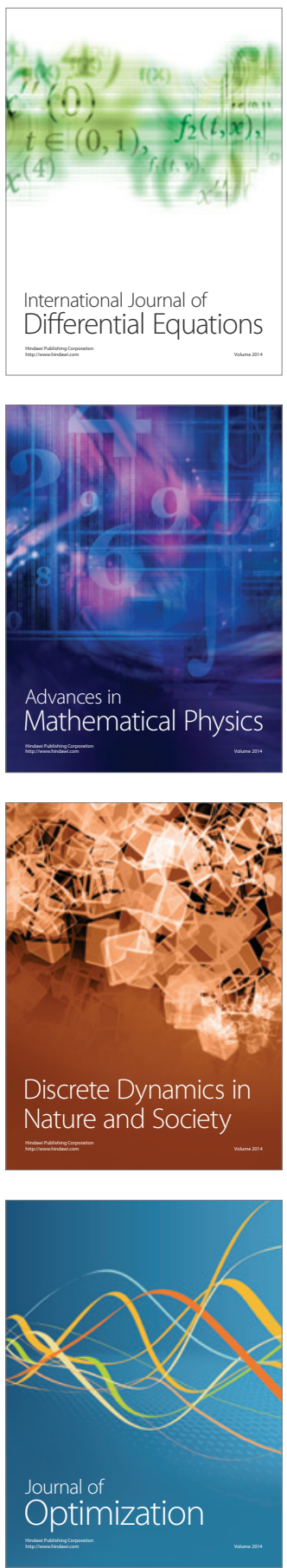